CLINICAL STUDY

\title{
Frequency and causes of adrenal crises over lifetime in patients with 21-hydroxylase deficiency
}

\author{
Nicole Reisch, Marina Willige, Denise Kohn ${ }^{1}$, Hans-Peter Schwarz ${ }^{2}$, Bruno Allolio ${ }^{4}$, Martin Reincke, \\ Marcus Quinkler ${ }^{3}$, Stefanie Hahner ${ }^{4}$ and Felix Beuschlein \\ Endocrine Research Unit, Medizinische Klinik und Poliklinik IV - Campus Innenstadt, Klinikum der Universität München, Endokrinologie, Ziemssenstrasse \\ 1, D-80336 München, Germany, ${ }^{1}$ Institute for Medical Information Sciences, Biometry and Epidemiology, Ludwig-Maximilians-Universität München, \\ Munich, Germany, ${ }^{2}$ Department of Endocrinology, von Haunersches Kinderspital, University Hospital Munich, Munich, Germany, ${ }^{3}$ Department of \\ Endocrinology, Universitätsmedizin Berlin Charité, Berlin, Germany and ${ }^{4}$ Department of Medicine I, Endocrinology and Diabetology, University Hospital \\ Würzburg, Würzburg, Germany \\ (Correspondence should be addressed to N Reisch; Email: nicole.reisch@med.uni-muenchen.de)
}

\begin{abstract}
Objective: To study adrenal crisis (AC) in patients with congenital adrenal hyperplasia due to classical 21-hydroxylase deficiency (21-OHD). AC was defined as an acute state of health impairment requiring i.v. glucocorticoid administration and hospital admission.

Design and methods: In a cross-sectional study with detailed retrospective assessment, AC was studied following two approaches: i) questionnaire based: 122 adult 21-OHD patients (50 men, 72 women, median age 35 years, range 18-69 years) completed a disease-specific questionnaire; and ii) patient chart based: charts of $6721-\mathrm{OHD}$ patients ( 32 males, 35 females, median age 31 years, range 20-66 years) were analyzed from diagnosis to last follow-up with regard to frequency and causes of AC since diagnosis.

Results: Evaluation of questionnaires revealed 257 ACs in 4456 patient years (py; frequency 5.8 crises/100 py), while patient charts documented 106 ACs in 2181 py (4.9 crises/100 py). The chartbased evaluation showed that gastrointestinal infections (29\%) and salt-wasting crisis (18\%) were the main causes of AC. In 14\%, the cause remained uncertain. There was no difference in the overall frequency of $\mathrm{AC}$ in males and females. AC mostly occurred during childhood, with more than $70 \%$ of $\mathrm{AC}$ in the first 10 years of life and one-third of $\mathrm{AC}$ in the first year of life. Still, $20 \%$ of cases of AC were observed in adults ( $>18$ years).

Conclusion: Our data demonstrate a significant risk of AC in patients with 21-OHD over lifetime. Specific age-adapted and repeated crisis prevention training may help to reduce morbidity due to AC in 21-OHD.
\end{abstract}

European Journal of Endocrinology 167 35-42

\section{Introduction}

Congenital adrenal hyperplasia (CAH) due to 21-hydroxylase deficiency (21-OHD) is an autosomal recessive metabolic disorder resulting in a lack of cortisol, an excess of adrenal androgens, and additionally in the salt-wasting (SW) form, diminished aldosterone secretion (1). It affects 1:11 000 newborns, approximately twothirds of which are suffering from the severe SW form (1). The unrecognized and thus untreated disease may lead to life-threatening SW crisis in the newborn period (2). Neonatal screening for classical 21-OHD, which is now routinely performed in most western countries, has significantly reduced the number of $S W$ crises in neonates and thereby mortality rates during infancy $(2,3,4,5)$. However, patients with classical CAH are at risk of lifethreatening adrenal crisis (AC) during their entire life $(6,7)$. In case of stress, the glucocorticoid replacement dose has to be increased in order to cover the increased demand in adrenal steroids. Inadequate or delayed glucocorticoid dose adjustment may result in severe metabolic crisis. Even though ACs are today largely preventable and rarely entailing fatal outcome, these events are still responsible for significant morbidity in $\mathrm{CAH}$. A major problem and challenge is to identify a risk situation in time in order to avoid AC, as first signs and presenting symptoms are variable and often nonspecific. To date, no data for $\mathrm{CAH}$ patients are available on the incidence, triggering causes, risk factors, or patients' awareness of AC. In fact, the current knowledge about $\mathrm{AC}$ in $\mathrm{CAH}$ is based on anecdotal evidence, personal clinical experience, and case reports. There are a few publications on AC in primary adrenal insufficiency (PAI) $(8,9,10,11,12)$ and in patients after adrenalectomy $(13,14)$, which in some aspects allow extrapolation to CAH. Both Addison's disease and CAH share the lack of 
cortisol (and aldosterone for the SW form) and thus the necessity of glucocorticoid dose adjustment in response to stress for the prevention of AC. However, PAI is an acquired disease in the vast majority of cases that mainly occurs in adults, leaving children and adolescents predominantly unaffected. Furthermore, it is unknown whether hyperandrogenism, due to 21-OHD, might modulate the frequency and severity of $\mathrm{AC}$ in $\mathrm{CAH}$ patients.

To gather further evidence on the clinical features of $\mathrm{AC}$ in $\mathrm{CAH}$, as well as age-dependent risk factors for $\mathrm{AC}$, we performed a cross-sectional study with detailed retrospective assessment of AC incidence in a cohort of 122 patients via a questionnaire and in 67 patients by investigation of patient charts. AC was defined as an acute state of health impairment that required i.v. glucocorticoid administration and hospital admission with no other discernible cause than AC or the risk of developing AC. We evaluated the patients' awareness and handling of $\mathrm{AC}$ as well as the professional management of AC by medical doctors. As patient surveillance and education about the recognition, prevention, and treatment of adrenal emergencies are the most effective and important strategies, this study demonstrates the need to improve targeted age- and disease-specific education of patients and/or their care givers.

\section{Subjects and methods}

\section{Patient population}

Adult patients with confirmed classical 21-OHD registered with the outpatient clinics at the University Hospitals of Munich, Würzburg and Charité Berlin, as well as members of the German self-help group for patients with $\mathrm{CAH}$, were asked to participate in the study and complete a disease-specific questionnaire. Additionally, charts of 21-OHD patients with regular and continuous hormonal follow-up from diagnosis until adulthood at the Endocrine outpatient clinic of the University Hospital of Munich, Germany (University Children's Hospital during childhood and adolescence, and Adult Endocrine Clinic of the University Hospital of Munich during adulthood) were studied according to documented AC. The parameters on biochemical, hormonal, and clinical endocrine control and causes of AC were extracted from the patients' medical records. The phenotype of 21-OHD was designated as either SW or simple virilizing (SV) 21-OHD, according to the use of mineralocorticoid substitution and renin concentration. AC was defined as an acute state of health impairment, which required i.v. glucocorticoid administration and hospital admission with no other discernible cause than AC or the risk of developing AC. Exclusion criteria for participation in this evaluation were: other diseases with impairment of adrenal function; severe general diseases (transaminases $>3 \times$ elevated levels, creatinine $>1.8 \mathrm{mg} / \mathrm{dl}(159.3 \mu \mathrm{mol} / \mathrm{l}))$; psychiatric diseases; or alcohol abuse. The study was approved by the local ethics committee, and all patients gave written informed consent.

\section{Disease-specific questionnaire}

Patients participating in the survey received a questionnaire asking for data on gender, age, age at diagnosis of 21-OHD, phenotype of 21-OHD, current medication, additional health problems, educational and occupational status, and the self-perceived influence of 21-OHD on activities of daily life. Frequency and causes of $\mathrm{AC}$ were documented, as well as patient equipment with an emergency card and emergency glucocorticoid medication. The questionnaire was designed in a multiple choice style. In addition, patients could add answers and comments as free text (modified from Hahner et al. (8)).

\section{Patient charts}

We studied medical records for the patients' history, the course and treatment of AC, and the biochemical, hormonal, and clinical findings associated with AC from the diagnosis of 21-OHD onward.

\section{Statistical analysis}

Statistical data of parameters that are not normally distributed is given as median (Q2) and first and third quartiles (Q1 and Q3). For group comparisons, statistical analyses were performed with the nonparametric Mann-Whitney $U$ test. Categorical variables were expressed as numbers and percentages and calculated by either $\chi^{2}$ test or Fisher's exact test. Twosided $P$ values are reported and $P$ values $<0.05$ were regarded significant. The self-reported and the documented occurrence of $\mathrm{AC}$ were determined as incidence rate with the number of AC per 100 patient years (py). A py was defined as 1 year of follow-up in the study. The effect of the following potential risk factors of $\mathrm{AC}$ have been investigated: phenotype and genotype of 21-OHD; sex; educational and occupational status; shift work; follow-up in university hospital or primary care center; prevalence of several concomitant diseases; change of glucocorticoid formula; and Prader stage at diagnosis. Causes of AC are reported in percentage of total numbers of AC. Statistical analysis was performed using SPSS for Windows 18.0 (SPSS, Inc., Chicago, IL, US) and SAS 9.2 (SAS Institute, Inc., Cary, NC, USA).

\section{Results}

\section{Patients}

In the questionnaire-based study, 122 patients with classical 21-OHD participated (42 from the outpatient clinic of the University Hospital of Munich, 25 from 
Table 1 Clinical characteristics of the patient cohorts showing the frequency of adrenal crisis (AC) in patients with classical 21-hydroxylase deficiency (21-OHD).

\begin{tabular}{|c|c|c|}
\hline Characteristics & Questionnaire & Patient chart \\
\hline Total number (n (\%)) & $122(100)$ & $67(100)$ \\
\hline Male $(n(\%))$ & $50(41)$ & $32(48)$ \\
\hline Female $(n(\%))$ & $72(59)$ & $35(52)$ \\
\hline $\mathrm{SW}(n(\%))$ & $71(58)$ & $46(69)$ \\
\hline SV $(n(\%))$ & $51(42)$ & $21(31)$ \\
\hline Experience of $A C$ & 70/122 (57) & 39/67 (58) \\
\hline $\begin{array}{l}\text { Age (years) at the time of } \\
\text { study (median (range)) }\end{array}$ & $35(18-69)$ & $31(20-66)$ \\
\hline $\begin{array}{l}\text { Total number of } \mathrm{AC} \\
\text { Number of } \mathrm{AC}(n(\%))\end{array}$ & 257 in $4456 \mathrm{PY}^{\mathrm{a}}$ & 106 in $2181 \mathrm{PY}^{\mathrm{b}}$ \\
\hline 0 & M: 19 (15.6); F: 33 (27.0) & M: 14 (20.9); F: 14 (20.9) \\
\hline 1 & M: 12 (9.8); F: 15 (12.3) & M: 5 (7.5); F: 12 (17.9) \\
\hline 2 & M: 11 (9.0); F: 3 (2.5) & M: 7 (10.4); F: 3 (4.5) \\
\hline 3 & $\mathrm{M}: 4$ (3.3); F: 5 (4.1) & $\mathrm{M}: 3$ (4.5); F: 1 (1.5) \\
\hline 4 & M: 0 (0); F: 4 (3.3) & M: 0 (0); F: 1 (1.5) \\
\hline$>4$ & M: 4 (3.3); F: 12 (9.8) & M: 3 (4.5); F: 4 (6.0) \\
\hline
\end{tabular}

SW, classical salt-wasting; SV, classical simple virilizing; PY, patient years; $\mathrm{M}$, male; $\mathrm{F}$, female.

a.8 AC/100 PY.

${ }^{\mathrm{b}} 4.9 \mathrm{AC} / 100 \mathrm{PY}$

the University Hospital of Charité Berlin, ten from the University Hospital of Würzburg, and 45 from the German self-help group for $\mathrm{CAH})$. In the chart-based study, 67 patient charts at the University Hospital of Munich with regular follow-up from diagnosis until 2011 were available for analysis. For 34 patients, both the questionnaire and the charts were available. The patients' characteristics are summarized in Table 1.

\section{Questionnaire}

According to the self-reported AC in the questionnaire, a total of 257 ACs in 4456 py (frequency 5.8 crises/ 100 py) occurred. From those, 57\% reported at least one AC. Of these, $61 \%$ had experienced more than one, and of those, $47 \%$ had four or more ACs (Table 1). Initial SW crisis that led to diagnosis accounted for more than half of the self-reported AC in 21-OHD patients (59\%), of whom $71 \%$ patients reported further crisis in their life.

According to patients' perception, AC was mostly triggered by infections with fever (29\%), particularly gastrointestinal infections (17\%) and urinary tract infections (5\%). Surgical interventions, emotional distress, and exhaustive physical exercise without adequate stress dosage caused $\mathrm{AC}$ according to the patients' self-reporting in 14,8 , and $7 \%$ respectively. Four percent of AC followed cessation of glucocorticoid medication by the patient and in $3 \%$ by the physician. Medical noncompliance as a cause of AC was also reported in 3\%. Other triggers were responsible for $7 \%$ of AC. In only $2 \%$ of $\mathrm{AC}$, the patients were not able to define any potential trigger.

AC was significantly more frequent in patients with SW 21-OHD ( $n=71,8.8$ crises $/ 100$ py) compared with the SV form $(n=51,2.5$ crises $/ 100$ py) $(P=0.002)$. $\mathrm{AC}$ was also more frequent in patients recruited via the self-help group $(n=45,6$ crises/100 py) compared with patients in regular care at the university hospitals $(n=77,5.7$ crises/100 py) $(P=0.049)$. There was no difference in AC frequency between males and females, or between different educational or occupational statuses. Overall, $89 \%(109 / 122)$ of the patients reported that they had previously adjusted their glucocorticoid dose during a stressful event. When asked in which situations they would increase their glucocorticoid medication, $85 \%$ of them reported that they would do so in case of infection with fever while $67 \%$ would increase the GC dosage in a perioperative setting, $22 \%$ in situations of psychological stress, and $13 \%$ when
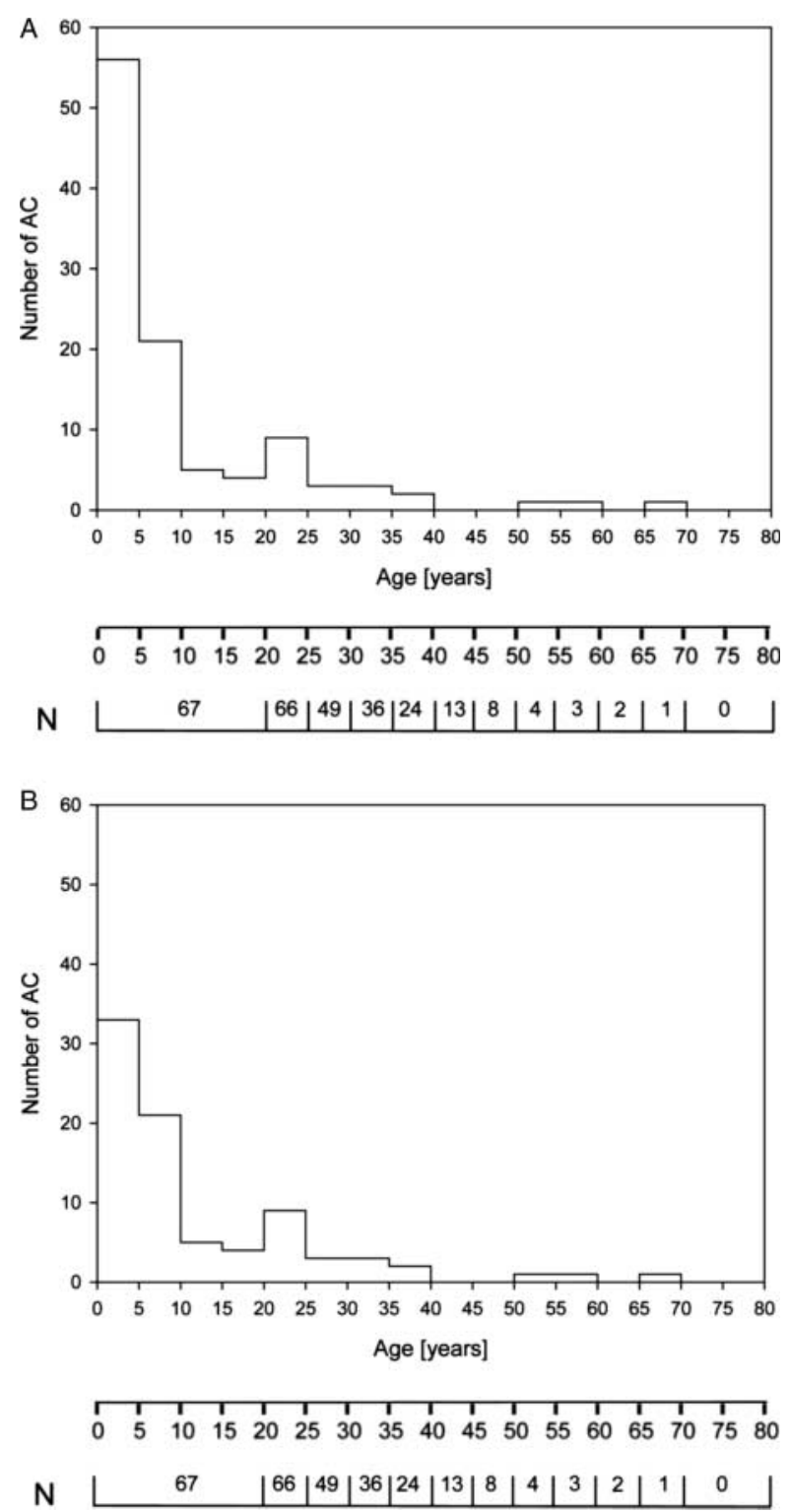

Figure 1 Histogram of the age-dependent distribution of $A C$ over lifetime with $(A)$ inclusion of initial SW crisis at first diagnosis and (B) after exclusion of initial SW crisis. 
doing exercise. Earlier experience of AC was associated with awareness of crisis prevention and glucocorticoid dose adaptation $(P=0.001)$. Eleven percent $(13 / 122)$ of patients had never adapted their glucocorticoid dose in stressful events, and 11 of these 13 patients had never experienced an $\mathrm{AC}$ in their entire lives. Ninety percent of patients declared themselves to be in possession of an emergency card and 22\% stated they had emergency medication (GC suppositories or GC vials for i.v. injection). The majority of patients felt that they coped 'very well' $(48 \%)$ or 'well' (31\%) with 21-OHD, whereas $5 \%$ of patients felt 'bad' and $2 \%$ 'very bad' in terms of coping with the disease. This subjective perception was not associated with frequency of $\mathrm{AC}(P=0.436)$.

\section{Patient charts}

In order to objectively confirm the self-reported triggers of $\mathrm{AC}$ as well as identifying risk factors and age-specific frequency of $\mathrm{AC}$, we evaluated the charts of 67 patients with 21-OHD from diagnosis onward from medical reports on AC. In 34 cases, both the questionnaire and the charts were available for analysis and showed an exact match of $\mathrm{AC}$ in $56 \%$, a deviation of \pm 1 in $18 \%$, and a deviation +1 in $26 \%$. The follow-up period documented in the charts was 27 years (median: Q1, 23 years; Q3, 28 years) in patients with SV form and 31 years (median: Q1, 24 years; Q3, 37 years) in patients with the SW form. In the medical files, a total of $106 \mathrm{ACs}$ in 2181 py were documented (4.9 AC/100 py). The majority of patients (58\%) experienced at least one $\mathrm{AC}$ in their lifetime, $33 \%$ more than one $\mathrm{AC}$, and $12 \%$ four or more AC (Table 1). Most ACs occurred in the first year of life $(33 / 106,31 \%)$, of which $67 \%$ of them were initial SW crises that led to diagnosis of 21-OHD (Fig. 1). If the initial SW crises were excluded from the analysis, $83 \mathrm{ACs}$ in 2181 py were evident, corresponding to $3.8 \mathrm{AC} / 100$ py. During ages $1-5,29$ ACs were documented $(27 \%)$, whereas in adulthood $(>18$ years), $20 \%$ of ACs were documented (Fig. 1). The median age at the time of $\mathrm{AC}$ was 4.5 years $(\mathrm{Q} 1,0.5$ years; $\mathrm{Q} 3$, 13.5 years). The SW phenotype was associated with $\mathrm{AC}$ $(P=0.001)$. Seventy-six percent of patients with genotype Null (15) (complete enzyme impairment; $n=14$ ) or A (almost complete enzyme impairment; $=15$ ) experienced AC compared with $43 \%$ of patients with genotype B (severe enzyme impairment, $<10 \%$ residual enzyme activity; $n=21$ ) (genotype Null and A vs B $P=0.018$ ).

In the data available, Prader stage was documented in 26 female patients, with 11 patients $(42 \%)$ with Prader stage 4, five patients each with Prader stages 2 and 3 (19\% each), one patient with Prader stages 2-3 (4\%), and four patients with Prader stages 3-4 (15\%). Prader stage was neither associated with incidence and frequency of $\mathrm{AC}$ nor associated with urinary tract infections. Figure 2A shows the distribution of documented triggers of AC with gastrointestinal infections as the most important underlying cause.
Patients suffering from allergies were at a higher risk of $\mathrm{AC}(P=0.007)$. Except for urinary tract infections with a clear bias toward females $(88 \%$, all before genital surgery), there was no gender difference for other preceding causes. The median age triggering an AC was 4 weeks for urinary tract infections $(\mathrm{Q} 1,1$ week; Q3, 8 months), 2 years for respiratory tract infections (Q1, 4 months; Q3, 5 years), 7 years for gastrointestinal infections (Q1, 3 years; Q3, 18 years), and 6 years for infections with fever (Q1, 1 year; Q3, 23 years) (Fig. 3).

The median duration of the hospital stay for $\mathrm{AC}$ was 5 days (Q1, 2 d; Q3, 27 d). Furthermore, AC was not associated with change of glucocorticoid formula, e.g. change from hydrocortisone to prednisolone or dexamethasone $(P=0.18)$. An association of $A C$ with any documented concomitant diseases could also be excluded (for all concomitant diseases, $P>0.06$ ).
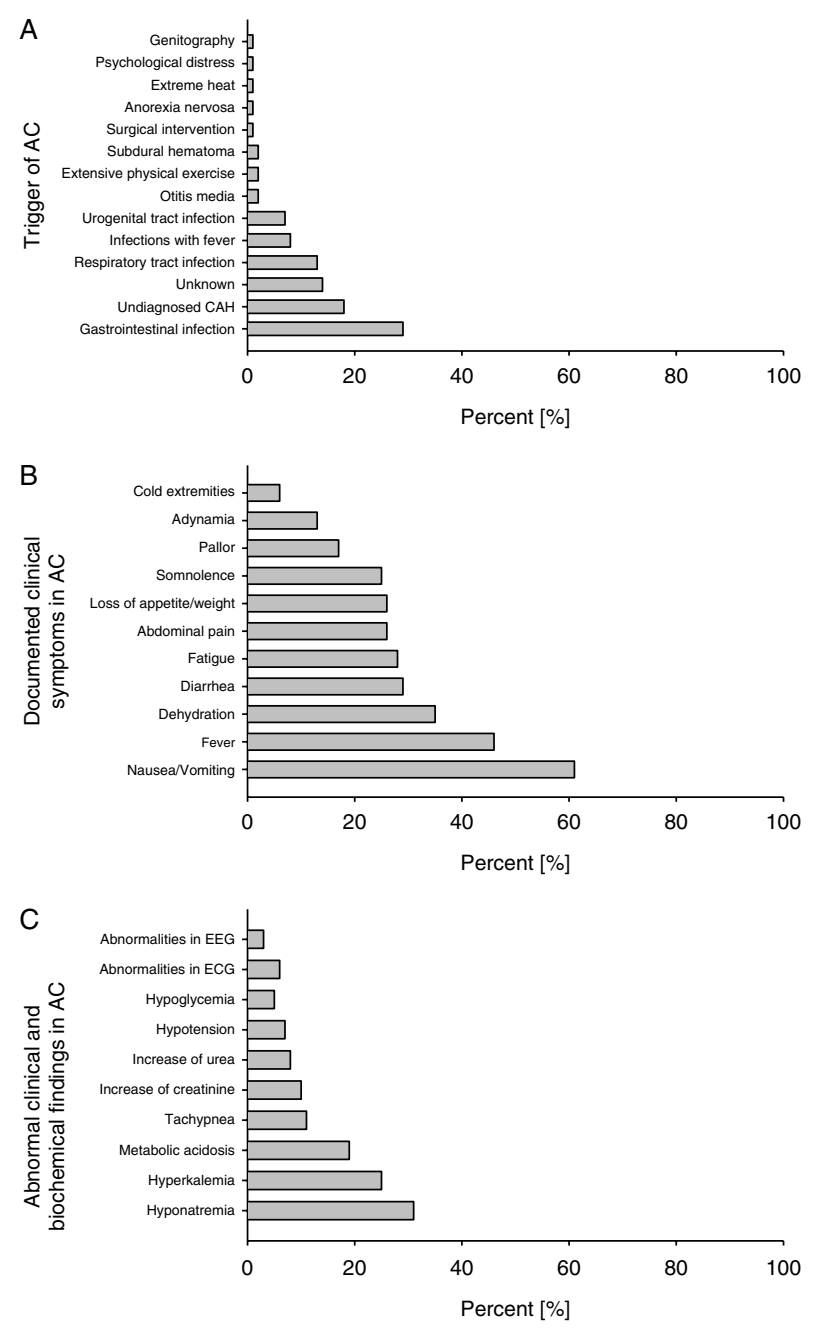

Figure 2 (A) Frequency of triggers of adrenal crisis (AC) $(n=106)$ according to the documentation in patient charts from 67 patients. (B) Documented clinical symptoms of AC in the patient charts of 67 patients with 106 documented ACs. (C) Documented abnormal clinical and biochemical findings in the patient charts of 67 patients with 106 documented ACs. 


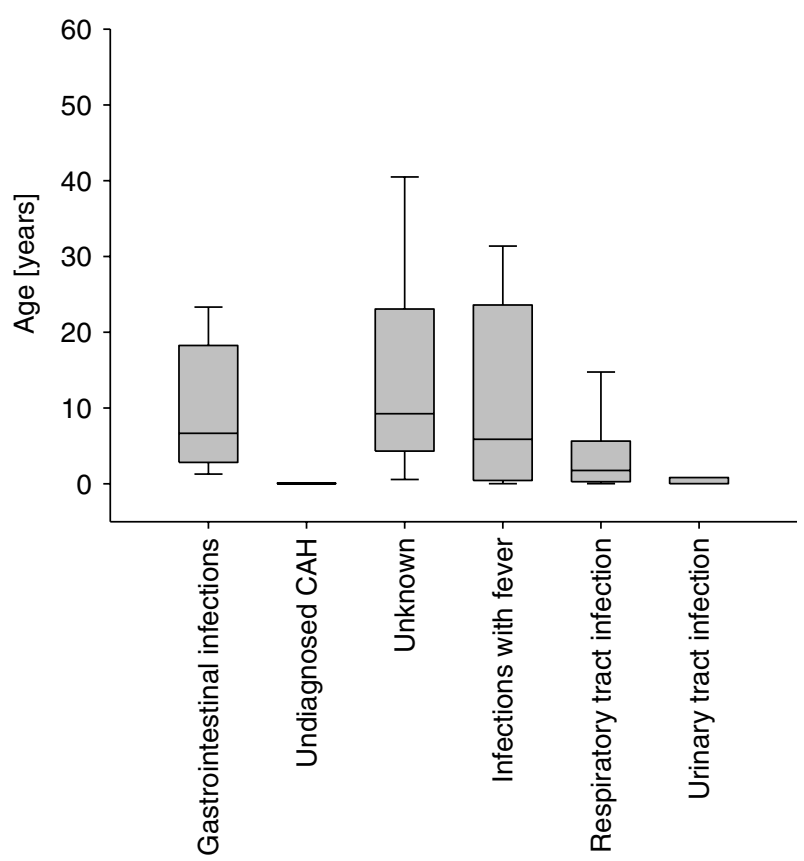

Figure 3 Age-dependent distribution of precipitating factors triggering adrenal crisis. The box plot provides the median age at $A C$ triggered by the specific underlying cause as well as the 10th, 25th (Q1), 75th (Q3), and 90th centiles.

Documented symptoms of AC are shown in Fig. 2B and documented clinical and biochemical findings are shown in Fig. 2C. The period between the patient/ parents noticing the first symptoms and AC was only 1 day (median, Q1, 0d; Q3, 3d, min. 0, max. 22d).

\section{Discussion}

In our cohort of classical 21-OHD patients, the incidence of $\mathrm{AC}$ was in a comparable range according to the patients' self-reporting and based on the patients' medical records. As would be expected, in the absence of prospective neonatal screening programs, in both the studies, the majority of metabolic crises could be attributed to SW crisis in newborns, which led to the diagnosis of $\mathrm{CAH}$. As neonatal screening was implemented nationwide in Germany in 2005 (16), and is currently carried out in most western countries, almost all these events will now be prevented. However, after correction for neonatal SW crisis, the frequency was still $4.9 \mathrm{AC} / 100$ py via self-reporting and $3.8 \mathrm{AC} / 100$ py according to documentation in the medical records. A significant proportion of these patients even experienced four or more AC (16\% selfreported, $12 \%$ chart based), underlining the necessity for better prevention strategies. In a recent analysis of patients with primary and secondary adrenal insufficiency, only a slightly higher number of ACs $(6.3 \mathrm{AC}$ per 100 py) has been observed (8). In contrast to adrenal insufficiency, AC in 21-OHD mostly occurs during infancy and childhood. Thus, repeated education and training of parents to improve awareness and prevention of AC is therefore essential. Interestingly, our study shows that there is a second peak of AC at the time when transition (age 18-25 years) from pediatric to adult endocrine care usually takes place. This highlights the necessity of good cooperation between pediatricians and endocrinologists during this vulnerable phase in order to prevent complications. It is interesting to note that in our cohort, puberty is obviously a less critical period for AC than the age between 20 and 25 years. Although causes for this finding will remain speculative, our clinical experience indicates that this, rather late, age is the time when patients start to take over self-management and self-responsibility of their disease from their parents. This may lead to temporary medical noncompliance reflected in a higher incidence of AC.

The slightly higher frequency of $\mathrm{AC}$ based on the questionnaire data might be explained by several reasons: events where glucocorticoids were administered intravenously in order to prevent $\mathrm{AC}$ might be regarded as crises by the patients but not documented as crisis in the charts. Secondly, a selection bias cannot be excluded as the questionnaire was distributed through a patient self-help group: patients who have already experienced AC may be more likely to join a self-help group and more motivated to participate in a survey than patients without any problems. In both instances, some degree of over-reporting is possible resulting in a potential overestimation of AC incidence. On the other hand, the chart-based study only included patients with regular follow-up from diagnosis onward (median follow-up 31 years), thus a bias toward compliant, wellcontrolled, and educated patients is likely. Currently, there are no comparative data of AC frequency in other populations available. In 34 patients, both the questionnaire and the charts were available for analysis and showed an exact match in $56 \%$ and a deviation of \pm 1 in $18 \%$, providing some evidence for the overall validity of these figures.

Our data provide some evidence that triggers for AC follow an age-related pattern. In infants, female babies were at particular risk for urinary tract infections before genital surgery. Even though Prader stage was not associated with frequency of AC, the most likely explanation for this finding is associated with genital malformations in females predisposing to urinary tract infections. Furthermore, pulmonary tract infections were main triggers of $\mathrm{AC}$ in early childhood, whereas gastrointestinal infections were the main cause throughout childhood and adolescence with a peak around the age of 6-7 years. Also in primary and secondary adrenal insufficiency, gastrointestinal infections have been shown to be the most important precipitating factors (8). However, as most ACs were associated with nausea and vomiting, and one-third of patients presented with diarrhea and abdominal 
symptoms, this might actually represent the clinical features of impending AC instead. Therefore, gastrointestinal infections may be overestimated as a trigger for AC. On the other hand, the incidence of acute gastrointestinal infections in the general population is quite high (17), indicating that only a smaller proportion of acute gastrointestinal infection in $\mathrm{CAH}$ patients in fact triggers the development of an $\mathrm{AC}$. Nevertheless, conditions of nausea and vomiting, irrespective of the underlying precipitator, appear to represent a special emergency situation in this patient group requiring rapid initiation of parenteral glucocorticoid replacement. According to our data, patients suffering from allergies were at higher risk for AC and increased caution should be taken in this cohort.

Our study also found that the median time from recognition of first symptoms to overt AC was only 1 day. This indicates that adrenal insufficiency develops rather suddenly or, at least, progresses rapidly as soon as it becomes clinically apparent. The availability of emergency medication is therefore essential. However, only $22 \%$ of patients indicated possession of an emergency kit. Similar to a study in patients with adrenal insufficiency, there was no association between the frequency of $\mathrm{AC}$ and the self-perceived level of information and coping with their disease. As previously suggested (8), regular and repeated structured training of patients with adrenal insufficiency, and their parents, should be performed similar to the training of patients with diabetes mellitus. A corresponding approach should also be mandatory for all patients (parents) with classical 21-OHD. This might help to significantly reduce morbidity and mortality from AC, especially in children. The study performed by Kovacs et al. (18) in five Central European countries found a mortality rate five times higher in patients diagnosed with SW CAH in infancy and childhood than for the general population. In most babies, deleterious outcome was associated with severe concurrent infections and/or sepsis; the suggested reason was inadequate adaption of glucocorticoid dose. Presumably, knowledge of prevention and treatment of $\mathrm{AC}$ has improved with time, thus reducing the current mortality rate in affected infants. The retrospective character of our investigation with data on AC during infancy being at least 18 years old is certainly a limitation of our study, because the incidence of AC in infancy has probably decreased in the intervening time.

Based on the results of our study, we ascertained four factors that contribute to development of $\mathrm{AC}$, and we suggest strategies to effectively prevent AC on each level (Fig. 4). The primary risk factor is the SW form of the disease, which is linked to specific genotypes of 21-OHD. Affected patients face a significantly higher risk for $\mathrm{AC}$ in the first weeks of life but the increased risk for $A C$ continues during their entire lifetime. Comparably, patients with PAI that also lack mineralocorticoids suffer more often from AC compared with patients with secondary adrenal insufficiency (8). Specific prevention strategies include newborn screening for classical 21-OHD in order to prevent SW crises in the first few weeks of life. Early diagnosis through neonatal screening programs with almost $100 \%$ capture rate enables early and adequate treatment of the disease in most western countries. Secondly, the quality of treatment and disease control influences the risk of AC. Routine follow-up should exclusively be provided in endocrine specialist care as ample experience with this rare disease is essential to strike the balance between over- and undertreatment in this patient cohort.

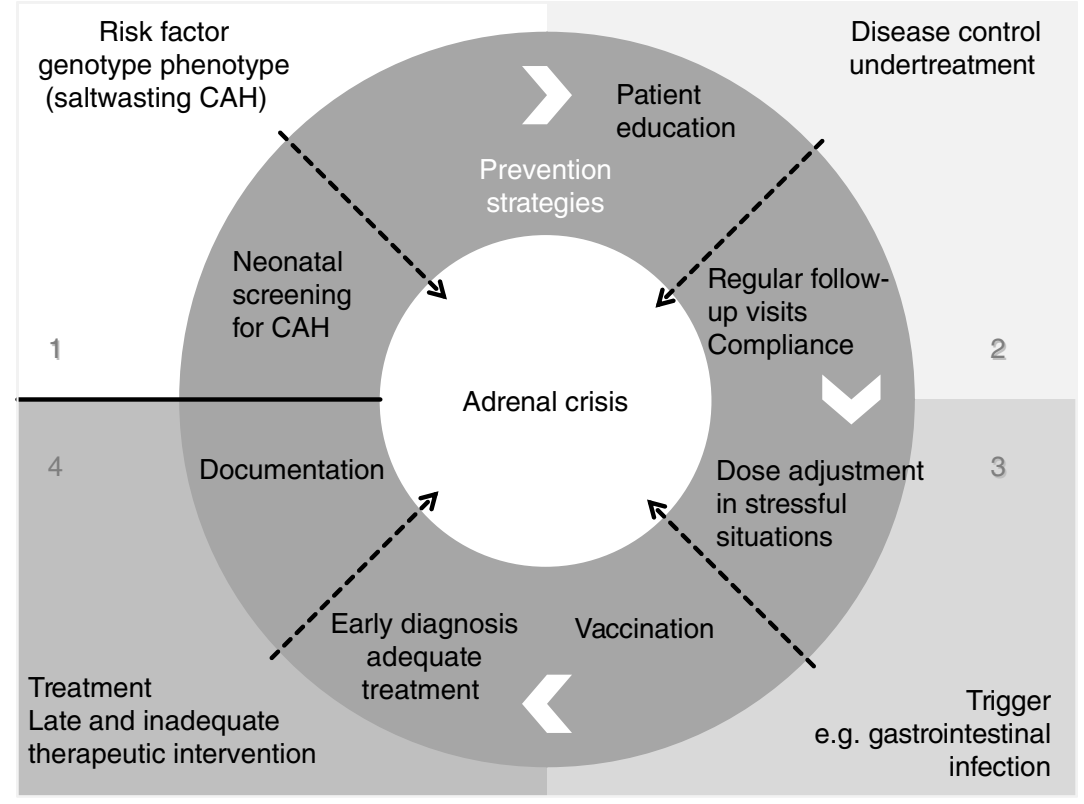

Figure 4 Schematic overview of causes leading to overt AC in classical 21-OHD and prevention strategies. 
The third aspect comprises the triggers of AC. As AC is often provoked by infection, broad vaccination, which is well tolerated, should be recommended to reduce avoidable infections (19). Finally, in beginning AC, the knowledge about the correct dose adjustment in stressful situations and the availability of emergency kits are critical. Patients and their parents need to be aware of the clinical symptoms of $\mathrm{AC}$ and the required treatment, including adaptation of the oral dose in cases of physical stress and also self-injection of hydrocortisone in more severe emergency situations. If no selfinjection kit is available in case of vomiting, we advise the patients to repeatedly take an increased oral dose (at least triple the dose) of hydrocortisone despite vomiting as some absorption of hydrocortisone takes place very quickly. But also health care professionals need to be continuously educated to be able to provide the correct diagnosis and treatment in a timely manner. Furthermore, we suggest improvement in the documentation of AC, which is often insufficient and incomplete. Although it is unlikely that interventional studies will ever be available on this topic, regular patient education is the most important management step to prevent $\mathrm{AC}$ and therefore should not be ignored.

In conclusion, our data demonstrate that $\mathrm{AC}$ is a clinically relevant problem in classical 21-OHD throughout lifetime and particularly during infancy and childhood. Underlying causes of AC are mainly infectious diseases with age-specific patterns. Raising awareness for the risk of $\mathrm{AC}$ and providing education on early prevention strategies of AC not only among patients and their relatives but also among primary care physicians may contribute to further reduce the frequency of AC and associated hospital admissions.

Some practical guidelines for patients to prevent AC are outlined below:

1. Always carry your steroid emergency card with you.

2. Situations that require glucocorticoid dose adjustment by yourself (triple dose of GC immediately):

a. Nausea with vomiting and diarrhea: in case no i.v. or i.m. injection is available, repeatedly take three times the oral dose of hydrocortisone despite vomiting, as some absorption of hydrocortisone takes place very quickly. See a physician without delay!

b. Intercurrent illness with fever $>38.5^{\circ} \mathrm{C}$ : triple hydrocortisone dose as long as body temperature continues to be elevated.

3. Situations where you may benefit from glucocorticoid dose adjustment:

a. Sustained psychological distress.

b. Extensive physical exercise: be cautious with glucocorticoid dose adjustment, as this has not been shown to be beneficial, but increase intake of sugar/carbohydrates (20).

4. Other situations: extreme heat - increase water and, in particular, salt intake.
5. Mineralocorticoid dose can be continued as usual, no increase of dose necessary.

6. Situations that require glucocorticoid dose adjustment by a physician:

a. Surgical intervention.

b. Shock, severe trauma, coma, and emergency surgery.

c. Continued vomiting and diarrhea.

\section{Declaration of interest}

The authors declare that there is no conflict of interest that could be perceived as prejudicing the impartiality of the research reported.

\section{Funding}

This work was supported by the Else Kröner-Fresenius-Stiftung (Grant 2010-EKES.29 to S Hahner and Grant 2011-EKMS.21 to N Reisch) and the European Community (Marie Curie European Reintegration Grant PERG-GA-2010-268270 to N Reisch).

\section{Acknowledgements}

The authors are grateful to Kathrin Zopf und Christiane Friedrich (both Charité Berlin, Germany) for help with the patients. They also thank Uschi Durant, Bianca Claße, and Doris Scharff from the German self-help group for CAH patients (AGS-Eltern- und Patienteninitiative e.V.) for their invaluable support by contacting the patients of this network and distributing the questionnaires.

\section{References}

1 Reisch N, Arlt W \& Krone N. Health problems in congenital adrenal hyperplasia due to 21-hydroxylase deficiency. Hormone Research in Paediatrics $2011 \quad \mathbf{7 6}$ 73-85. (doi:10.1159/ 000327794)

2 White PC. Neonatal screening for congenital adrenal hyperplasia. Nature Reviews. Endocrinology 20095 490-498. (doi:10.1038/ nrendo.2009.148)

3 Grosse SD \& Van Vliet G. How many deaths can be prevented by newborn screening for congenital adrenal hyperplasia? Hormone Research 200767 284-291. (doi:10.1159/000098400)

4 Cavarzere P, Camilot M, Teofoli F \& Tato L. Neonatal screening for congenital adrenal hyperplasia in North-Eastern Italy: a report three years into the program. Hormone Research 200563 180-186. (doi:10.1159/000085021)

5 Steigert M, Schoenle EJ, Biason-Lauber A \& Torresani T. High reliability of neonatal screening for congenital adrenal hyperplasia in Switzerland. Journal of Clinical Endocrinology and Metabolism 200287 4106-4110. (doi:10.1210/jc.2002-012093)

6 Merke DP \& Bornstein SR. Congenital adrenal hyperplasia. Lancet 2005365 2125-2136. (doi:10.1016/S0140-6736(05)66736-0)

7 Speiser PW, Azziz R, Baskin LS, Ghizzoni L, Hensle TW, Merke DP, Meyer-Bahlburg HF, Miller WL, Montori VM, Oberfield SE, Ritzen M \& White PC. Congenital adrenal hyperplasia due to steroid 21-hydroxylase deficiency: an Endocrine Society clinical practice guideline. Journal of Clinical Endocrinology and Metabolism 201095 4133-4160. (doi:10.1210/jc.2009-2631)

8 Hahner S, Loeffler M, Bleicken B, Drechsler C, Milovanovic D, Fassnacht M, Ventz M, Quinkler M \& Allolio B. Epidemiology of adrenal crisis in chronic adrenal insufficiency: the need for new prevention strategies. European Journal of Endocrinology 2010162 597-602. (doi:10.1530/EJE-09-0884) 
9 White K \& Arlt W. Adrenal crisis in treated Addison's disease: a predictable but under-managed event. European Journal of Endocrinology 2010162 115-120. (doi:10.1530/EJE-09-0559)

10 Omori K, Nomura K, Shimizu S, Omori N \& Takano K. Risk factors for adrenal crisis in patients with adrenal insufficiency. Endocrine Journal 200350 745-752. (doi:10.1507/endocrj.50.745)

11 Arlt W \& Allolio B. Adrenal insufficiency. Lancet 2003361 1881-1893. (doi:10.1016/S0140-6736(03)13492-7)

12 Braatvedt GD, Newrick PG \& Corrall RJ. Patients' self administration of hydrocortisone. BMJ 1990301 1312. (doi:10.1136/ bmj.301.6764.1312)

13 Nagesser SK, van Seters AP, Kievit J, Hermans J, Krans HM \& van de Velde CJ. Long-term results of total adrenalectomy for Cushing's disease. World Journal of Surgery 200024 108-113. (doi:10.1007/s002689910020)

14 Thompson SK, Hayman AV, Ludlam WH, Deveney CW, Loriaux DL \& Sheppard BC. Improved quality of life after bilateral laparoscopic adrenalectomy for Cushing's disease: a 10-year experience. Annals of Surgery $2007 \mathbf{2 4 5}$ 790-794. (doi:10.1097/01.sla. $0000251578.03883 .2 \mathrm{f})$

15 Krone N, Braun A, Roscher AA, Knorr D \& Schwarz HP. Predicting phenotype in steroid 21-hydroxylase deficiency? Comprehensive genotyping in 155 unrelated, well defined patients from Southern Germany. Journal of Clinical Endocrinology and Metabolism 200085 1059-1065. (doi:10.1210/jc.85.3.1059)

16 Harms E \& Olgemoller B. Neonatal screening for metabolic and endocrine disorders. Deutsches Ärzteblatt International $2011 \mathbf{1 0 8}$ 11-21 (quiz 22).
17 Baumann-Popczyk A, Sadkowska-Todys M, Rogalska J \& Stefanoff P. Incidence of self-reported acute gastrointestinal infections in the community in Poland: a population-based study. Epidemiology and Infection 201119 1-12. (doi:10.1017/ S0950268811001853)

18 Kovacs J, Votava F, Heinze G, Solyom J, Lebl J, Pribilincova Z, Frisch H, Battelino T \& Waldhauser F. Lessons from 30 years of clinical diagnosis and treatment of congenital adrenal hyperplasia in five middle European countries. Journal of Clinical Endocrinology and Metabolism 200186 2958-2964. (doi:10.1210/jc.86.7. 2958)

19 Weiss M, Dorr HG, Brandmaier R, Schwarz HP \& Belohradsky BH. Vaccine tolerance in steroid substituted patients with congenital adrenal hyperplasia. European Journal of Medical Research 19972 290-292.

20 Weise M, Drinkard B, Mehlinger SL, Holzer SM, Eisenhofer G, Charmandari E, Chrousos GP \& Merke DP. Stress dose of hydrocortisone is not beneficial in patients with classic congenital adrenal hyperplasia undergoing short-term, high-intensity exercise. Journal of Clinical Endocrinology and Metabolism 200489 3679-3684. (doi:10.1210/jc.2003-032051)

Received 22 February 2012

Revised version received 4 April 2012

Accepted 18 April 2012 